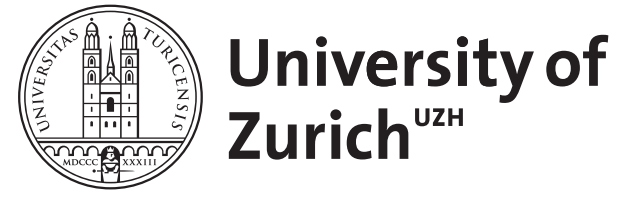

\title{
Cabled Wilson loops in BF theories
}

\author{
Cattaneo, A S
}

\begin{abstract}
A generating function for cabled Wilson loops in three-dimensional BF theories is defined, and a careful study of its behavior for vanishing cosmological constant is performed. This allows an exhaustive description of the unframed knot invariants coming from the pure BF theory based on $\mathrm{SU}(2)$, and, in particular, it proves a conjecture relating them to the Alexander-Conway polynomial. () 1996 American Institute of Physics.
\end{abstract}

DOI: https://doi.org/10.1063/1.531595

Posted at the Zurich Open Repository and Archive, University of Zurich

ZORA URL: https://doi.org/10.5167/uzh-22541

Journal Article

Published Version

Originally published at:

Cattaneo, A S (1996). Cabled Wilson loops in BF theories. Journal of Mathematical Physics, 37(8):36843703.

DOI: https://doi.org/10.1063/1.531595 


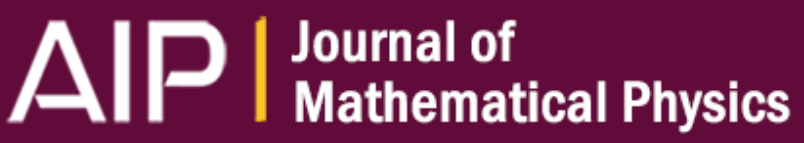

\section{Cabled Wilson loops in BF theories}

Alberto S. Cattaneo

Citation: Journal of Mathematical Physics 37, 3684 (1996); doi: 10.1063/1.531595

View online: http://dx.doi.org/10.1063/1.531595

View Table of Contents: http://scitation.aip.org/content/aip/journal/jmp/37/8?ver=pdfcov

Published by the AIP Publishing

\section{Articles you may be interested in}

Superspace version of BF theories

AIP Conf. Proc. 1444, 207 (2012); 10.1063/1.4715421

Light-like Wilson Loops and Cusp Anomalous Dimensions in Nonconformal Gauge Theories AIP Conf. Proc. 1031, 159 (2008); 10.1063/1.2972003

Wilson loop and dimensional reduction in non-commutative gauge theories

AIP Conf. Proc. 607, 313 (2002); 10.1063/1.1454390

Poisson brackets of normal-ordered Wilson loops

J. Math. Phys. 40, 1870 (1999); 10.1063/1.532838

Poisson brackets of Wilson loops and derivations of free algebras

J. Math. Phys. 37, 637 (1996); 10.1063/1.531433

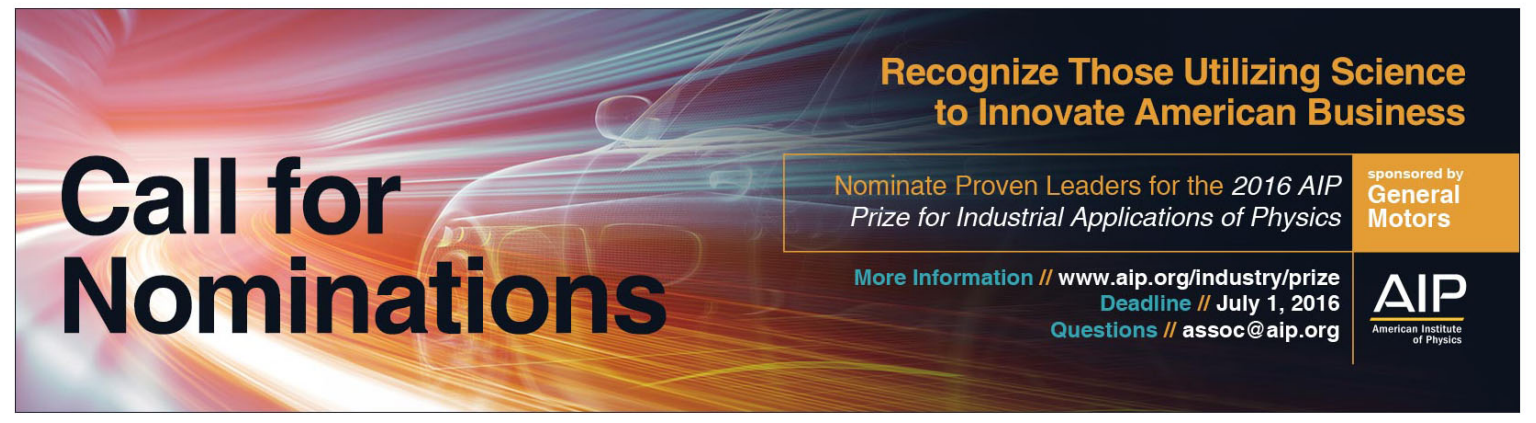




\title{
Cabled Wilson loops in BF theories
}

\author{
Alberto S. Cattaneo ${ }^{\text {a) }}$ \\ Lyman Laboratory of Physics, Harvard University, Cambridge, Massachusetts 02138
}

(Received 8 February 1996; accepted for publication 29 April 1996)

A generating function for cabled Wilson loops in three-dimensional BF theories is defined, and a careful study of its behavior for vanishing cosmological constant is performed. This allows an exhaustive description of the unframed knot invariants coming from the pure BF theory based on SU(2), and, in particular, it proves a conjecture relating them to the Alexander-Conway polynomial. (C) 1996 American Institute of Physics. [S0022-2488(96)00308-8]

\section{INTRODUCTION}

In Ref. 1, a relation was conjectured between the Alexander-Conway polynomial and the v.e.v. (vacuum expectation value) of a newly proposed observable for the TQFT (topological quantum field theory) known as BF theory ${ }^{2}$ (or as "pure" BF theory, to distinguish it from its generalization "with a cosmological term"). The main purpose of this paper is to clarify that conjecture and to prove it in the case of SU(2), although in a slightly different form. To do so, however, we also perform a careful study of BF theories, both pure and with a cosmological term, introducing new observables that describe cabled Wilson loops.

The BF theories are interesting from both the mathematical and the physical points of view: They are TQFTs that, in principle, can be defined in any dimension (see Ref. 3 and references therein). They describe quantum gravity in three dimensions ${ }^{4}$ and could be useful for the study of four-dimensional quantum gravity in the approach of Ref. 5. Moreover, they are related to YangMills theories in the weak-coupling limit, where they could be used to describe a "topological phase" ${ }^{\prime 6}$ (see also Ref. 7 for a related approach). Finally, hitherto only in the Abelian case, they have been used to study many-body systems. ${ }^{8}$

On the other side, the interest for the Alexander-Conway polynomial comes from the fact that it has always played a different role than the other knot invariants, being the only one that, hitherto, has been described by the methods of the traditional algebraic topology; yet it lacked a TQFT description (and Ref. 1 was an attempt to fill this gap).

Indeed, the Chern-Simons theory describes the knot invariants related to the $q$-deformation of a classical group $G$ in terms of v.e.v.s of Wilson loops (i.e., traces of $G$-holonomies along the knots); ${ }^{9}$ yet the $q=1$ case, i.e., the Alexander-Conway polynomial, does not correspond to any acceptable value of the Chern-Simons coupling constant $k$.

In the case of $\mathrm{SU}(2)$, an important improvement was given by the Melvin-Morton conjecture $^{10}$ (proved in Ref. 11), which states that the inverse of the Alexander-Conway polynomial appears in the $q-1$ expansion of the colored Jones function. Rozansky ${ }^{12}$ was then able to prove the same result in the framework of the Chern-Simons theory; more precisely, he showed that the inverse of the Alexander-Conway polynomial is recovered by a saddle-point computation in the limit $k \rightarrow \infty$.

In Ref. 3, an equivalence between the v.e.v.s of the Chern-Simons theory and of the BF theory with cosmological constant $\kappa=1 /(2 k)$ was shown. The $k \rightarrow \infty$ limit (i.e., the $q=1$ case) is then simply described by removing the cosmological term, thus obtaining the pure BF theory.

In this paper we show that the trace in the fundamental representation of the pure-BF-theory observable (31), first introduced in Ref. 1 , is related to the $\kappa \rightarrow 0$ limit of a newly proposed

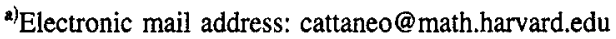


observable, (22), for the BF theory with a cosmological term. In the framework of the ChernSimons theory, this observable corresponds to the "exponential" of a holonomy; so its trace is the generating function for cabled Wilson loops, and the v.e.v. of this trace is the generating function for cabled knot invariants (as suggested by the results of Ref. 13).

Since the colored Jones functions are related to the cabled knot invariants, we are able to connect the $q-1$ expansion of the former to the pure-BF-theory knot invariant. By the MelvinMorton conjecture, we conclude that the latter is related to the Alexander-Conway polynomial, the precise relation being (49). Our proof, however, can also go the other way; i.e., should one directly prove a relation between the pure BF theory and the Alexander-Conway polynomial, then we would have a further proof of the Melvin-Morton conjecture.

Our results are not limited to the trace in the fundamental representation of a particular observable for pure BF theory. In fact, we are able to prove that, in the standard framing, this observable is the most general one can consider (see Theorem 1 and Corollary 1). Moreover, we show how to compute the higher-representation knot invariants [see (34) and (35)], and also evaluate the limit as the dimension of the representation goes to infinity [see (37) and (40)]. Thus, we have a complete description of the unframed knot invariants coming from the pure BF theory based on SU(2) (see Theorem 2). The case of links has not been considered yet.

The paper is organized as follows: In Sec. II, we describe the colored Jones functions and introduce their generating function. In Sec. III, we recall the property of the BF theory with a cosmological term and define a generating function for cabled Wilson loops. In Sec. IV, we discuss the pure BF theory and prove Theorem 1 and Corollary 1 . In Scc. V, we consider the limit for vanishing cosmological constant and prove Theorem 2. We conclude our work in Sec. VI, comparing our present results with those obtained perturbatively in Ref. 1. For the sake of clarity, we have put all the cumbersome computations into some appendices, to which we shall refer in the text.

\section{THE COLORED JONES FUNCTION}

The colored Jones function, $J_{d}(C ; h)$, is defined in Ref. 10 as the invariant of the knot $C$ obtained using the irreducible $\mathrm{SU}(2)_{q}$-module $\left(q=e^{h}\right)$ of dimension $d$. The main properties of its expansion as a rational power series (here and in the following, the notation of Ref. 11 is used),

$$
J_{d}(C ; h)=\sum_{m=0}^{\infty} \widetilde{J}_{m}(C ; d) h^{m}=d \sum_{j, m=0}^{\infty} b_{j m}(C)(d-1)^{j} h^{m}
$$

were stated in Ref. 10:

(i) The functions $\widetilde{J}_{m}(C ; d)$ are odd in $d$. This means that (the analytic continuation of) $J_{d}(C ; h)$ itself is odd:

$$
J_{-d}(C ; h)=-J_{d}(C ; h) .
$$

[This of course implies that the coefficients $b_{j m}$ in (1) are not completely independent.]

(ii) These functions are actually polynomials of degree not exceeding $2 m+1$.

(iii) In the hypothesis that the standard framing for $\mathrm{C}$ is chosen, the degree of $\widetilde{J}_{m}$ cannot exceed $2 m-1$.

In the same hypothesis of standard framing, two conjectures were also stated in Ref. 10:

(i) The degree of $\widetilde{J}_{m}$ cannot actually exceed $m+1$, or in other words, $b$. in (1) is an upper triangular matrix.

$$
b_{j m}(C)=0, \quad \text { if } j>m \text {. }
$$

(ii) The diagonal elements of $b$.. are related to the Alexander-Conway polynomial; viz., if one defines the Melvin-Morton function as 


$$
J J(C ; \hbar)=\sum_{m=0}^{\infty} b_{m m}(C) \hbar^{m}=\lim _{d \rightarrow \infty, h \rightarrow 0, d h=\hbar} \frac{J_{d}(C ; h)}{d}
$$

[the limil existing because of (3)], then

$$
\hbar J J(C ; \hbar)=\frac{z}{\Delta(C ; z)}, \quad z=e^{\hbar / 2}-e^{-\hbar / 2}
$$

where $\Delta(C ; z)$ is the Alexander-Conway polynomial with the normalization $\Delta(O ; z)=1$, where $O$ is the unknot, and skein relation

$$
\Delta\left(C_{+} ; z\right)-\Delta\left(C_{-} ; z\right)=z \Delta\left(C_{0} ; z\right)
$$

These two conjectures have been given a functional-integral proof by Rozansky. ${ }^{12}$ In particular he proved that the Melvin-Morton function, (4), is related to the large- $k$ limit of the ChernSimons theory, so it can be computed in saddle-point approximation.

Eventually, Bar-Natan and Garoufalidis ${ }^{11}$ gave a mathematical proof of (3) and (5) on the level of weight systems.

\section{A. The generating function for colored Jones functions}

In the following sections, the generating function

$$
f(C ; x, h)=\sum_{d=1}^{\infty} \frac{x^{d}}{d !} J_{d}(C ; h),
$$

with $x \in \mathrm{C}$, will be needed; so it is useful to anticipate here some of its properties. These are better clarified if one introduces $\tilde{f}$ defined by

$$
f(C ; x, h)=x e^{x} \widetilde{f}(C ; x, h) .
$$

In fact, the expansion of $\tilde{f}$ as a rational power series,

$$
\widetilde{f}(C ; x, h)=\sum_{j, m=0}^{\infty} \widetilde{b}_{j m}(C) x^{j} h^{m},
$$

shares the same properties as the expansion (1) of $J_{d}(C ; h)$ (viz., see Appendix B). One can prove that

$$
b . \text { upper triangular } \Leftrightarrow \tilde{b} \text {.. upper triangular. }
$$

Thus, (3) implies that $\widetilde{b} .$. is actually an upper triangular matrix,

$$
\tilde{b}_{j m}(C)=0, \text { if } j>m,
$$

and that $\widetilde{f}$ has a well-defined limit for $|x| \rightarrow \infty, h \rightarrow 0$, and $x h=\hbar$ kept fixed. In Appendix B, it is shown that

$$
\lim _{|x| \rightarrow \infty, h \rightarrow 0, x h=\hbar} \widetilde{f}(C ; x, h)=J J(C ; \hbar),
$$

and that, in general, for any $n \geqslant 0$, 


$$
\lim _{|x| \rightarrow \infty, h \rightarrow 0, x h=\hbar}\left(x \frac{\partial}{\partial x}\right)^{n} \widetilde{f}(C ; x, h)=\left(\hbar \frac{d}{d \hbar}\right)^{n} J J(C ; \hbar) .
$$

\section{THE BF THEORY WITH A COSMOLOGICAL TERM}

From the field-theoretical point of view, the colored Jones function is defined as the knot invariant obtained from the v.e.v. of a suitable observable, the Wilson loop, in the Chern-Simons theory ${ }^{9}$ (actually, the general case of a compact simple Lie group is discussed there).

For the aims of this paper, however, it is more convenient to consider the formulation given in terms of the BF theory with a cosmological term, which is equivalent $t^{3.14}$ to the previous one.

We will consider here only the case of a single knot $C$ imbedded in $S^{3}$ and study its invariants associated to the group SU(2); viz., we consider the SU(2)-principal bundle $P \rightarrow S^{3}$, and define the action

$$
\widetilde{S_{\mathrm{BF}}}(\kappa)=\frac{1}{2 \pi} \int_{S^{3}} \operatorname{Tr}\left(B \wedge F+\frac{\kappa^{2}}{3} B \wedge B \wedge B\right),
$$

where $F$ is the curvature two-form of the connection $A, B$ is a form in $\Omega^{1}\left(S^{3}\right.$,ad $\left.P\right)$, and the parameter $\kappa$ is called the cosmological constant (this name comes from the quantum-gravity interpretation of $\left.(13)^{4}\right)$.

Then, given a knot $C$ and a base point $x_{0} \in C$, we consider the observable

$$
\Gamma\left(C, x_{0} ; \kappa\right):=\operatorname{Hol}_{x_{0}}(A+\kappa B ; C)=\sum_{n=0}^{\infty} \kappa^{n} \gamma_{n}\left(C, x_{0}\right),
$$

where $\kappa$ is the same cosmological constant as in (13), $\operatorname{Hol}_{x_{0}}(A+\kappa B ; C)$ denotes the holonomy of the connection $A+\kappa B$ along the knot $C$ with base point $x_{0}$, and the $\gamma$ are functionals of $A$ and $B$ obtained by Taylor expanding the previous holonomy.

Notice that the holonomy of a connection $A$ along a curve $C$, open or closed, can be written as

$$
\operatorname{Hol}(A ; C)=P \exp \left(\int_{C} A\right)
$$

where $P$ denotes path ordering. Thus, by (14) and (15), the $\gamma$ s turn out to be ${ }^{3,14}$ iterated Chen integrals of the form

$$
\gamma_{n}\left(C, x_{0}\right):=\oint_{\left(C ; x_{0}\right)} \underbrace{\hat{B} \cdots \hat{B}}_{n \text { times }} \operatorname{Hol}_{x_{0}}(A ; C),
$$

where the iterated integral $\int_{a}^{b} \omega_{1} \cdots \omega_{2} \cdots \omega_{n}$ of $n$ one-forms $\left\{\omega_{i}\right\}_{i=1, \ldots, n}$ is given by the formula $\int_{a<x_{1}<\cdots<x_{n}<b} \omega_{1}\left(x_{1}\right) \wedge \omega_{2}\left(x_{2}\right) \wedge \cdots \wedge \omega_{n}\left(x_{n}\right)$. The $\operatorname{su}(2)$-valued one-form $\hat{B}$ is defined as

$$
\hat{B}(x):=\operatorname{Hol}_{x_{0}}^{x} B(x)\left[\mathrm{Hol}_{x_{0}}^{x}\right]^{-1},
$$

with the holonomies computed along the portions of the knot $C$ going from the base point $x_{0}$ to the running points $x$.

The observable $\Gamma$ in (14) is invariant under a gauge transformation of the connection $A-\kappa B$, while, under the gauge transformation 


$$
A+\kappa B \rightarrow g(A+\kappa B) g^{-1}+g d g^{-1},
$$

one has

$$
\Gamma\left(C, x_{0} ; \kappa\right) \rightarrow g\left(x_{0}\right) \Gamma\left(C, x_{0} ; \kappa\right) g\left(x_{0}\right)^{-1} .
$$

Thus, taking the trace of $\Gamma$ gives a gauge invariant observable that is, moreover, independent of $x_{0}$. The knot invariant given by the v.e.v. of this observable, wrt the (normalized) Gibbs weight $\exp \left[i S_{\mathrm{BF}}(\kappa)\right]$, can be recognized by exploiting the relation with the Chern-Simons theory, ${ }^{3}$ viz.,

$$
J_{d}(C ; h)=\left\langle\operatorname{Tr}_{(d-1) / 2} \Gamma\left(C, x_{0} ; \kappa\right)\right\rangle_{\mathrm{BF}, \kappa}, \quad h=4 \pi i \kappa,
$$

where $\operatorname{Tr}_{s}$ is the trace in the irreducible representation of spin $s$. In this framework, (2) is an immediate consequence of (A8).

Notice, eventually, that the observable $\Gamma$ can be decomposed into its even $\left(\Gamma_{0}\right)$ and odd $\left(\kappa \Gamma_{1}\right)$ parts,

$$
\Gamma\left(C, x_{0} ; \kappa\right)=\Gamma_{0}\left(C, x_{0} ; \kappa\right)+\kappa \Gamma_{1}\left(C, x_{0} ; \kappa\right),
$$

and that both of them are good observables for the BF theory with a cosmological term. ${ }^{14}$

\section{A. The BF-theory generating function}

The behavior (19) of the observable $\Gamma$ under a gauge transformation of the connection $A+\kappa B$ shows that all of the traces of powers of $\Gamma$ are gauge invariant; so we can consider the knot invariants given by their v.e.v.s, or as will be clear in Sec. $V$, their generating function,

$$
\begin{gathered}
E(C ; x, h)=\sum_{n=0}^{\infty} \frac{x^{n}}{n !}\left\langle\operatorname{Tr}_{1 / 2} \Gamma\left(C, x_{0} ; \kappa\right)^{n}\right\rangle_{\mathrm{BF}, \kappa}=\left\langle\operatorname{Tr}_{1 / 2} \exp \left[x \Gamma\left(C, x_{0} ; \kappa\right)\right]\right\rangle_{\mathrm{BF}, \kappa}, \\
h=4 \pi i \kappa
\end{gathered}
$$

with $x \in \mathrm{C}$. We consider only the fundamental representation here, for the discussion in Appendix A shows that considering any other representation does not give further content of information. Notice that the rightmost term in (22) is only formal since no addition is defined in the group. However, this notation usefully reminds us that $E$ has the formal properties of an exponential. Notice that, since $\Gamma$ is a holonomy, one has

$$
\Gamma\left(C, x_{0} ; \kappa\right)^{n}=\Gamma\left(n C, x_{0} ; \kappa\right),
$$

where by $n C$ we denote the $n$th cabling of the knot $C$; thus, $E(C ; x, h)$ is the generating function for cabled knot invariants. In the framework of the Chern-Simons theory, $\Gamma$ is replaced by the holonomy of the connection $A$; thus, $E$ can also be seen as the v.e.v. of the generating function for cabled Wilson loops.

By using the formulas in Appendix A, one can show that there is a simple relation with the generating function for colored Jones functions defined in (6). Actually by (A6), one can express $\operatorname{Tr}_{1 / 2} \Gamma^{n}$ in terms of the traces of $\Gamma$ in the representations of spin $n / 2$ and $n / 2-1$. By (20), it then follows that

$$
E(C ; x, h)=\sum_{n=0}^{\infty} \frac{x^{n}}{n !}\left[J_{n+1}(C ; h)-J_{n-1}(C ; h)\right],
$$

where, according to (A8), $J_{1}=1, J_{0}=0, J_{-1}=-1$. 
By (6) and (24), and also noting that $E(C ; 0, h)=2$ and $\partial_{x} f(C ; 0, h)=J_{1}(C ; h)=1$, one obtains

$$
E(C ; x, h)=1+\partial_{x} f(C ; x, h)-\int_{0}^{x} d \xi f(C ; \xi, h) \text {. }
$$

\section{THE PURE BF THEORY}

The BF theory has the nice property that its limit for vanishing cosmological constant (corresponding to the $k \rightarrow \infty$ limit in the Chern-Simons theory) is still represented by a TQFT known as pure $\mathrm{BF}$ theory, ${ }^{2,15}$ whose action reads

$$
S_{\mathrm{BF}}=\frac{1}{2 \pi} \int_{S^{3}} \operatorname{Tr}(B \wedge F)
$$

It is immediately seen that $S_{\mathrm{BF}}$ is invariant under gauge transformations

$$
A \rightarrow g A g^{-1}+g d g^{-1}, \quad B \rightarrow g B g^{-1},
$$

as well as under $B$-transformations

$$
A \rightarrow A, \quad B \rightarrow B+d_{A} \psi
$$

Here $g$ is a map from $S^{3}$ to the group, while $\psi$ is a form in $\Omega^{0}\left(S^{3}\right.$,ad $\left.P\right)$. The action (26) is invariant under (28) owing to the Bianchi identity.

The good observables for the pure BF theory are of course obtained by $\Gamma_{0}\left(C, x_{0} ; \kappa\right)$ and $\Gamma_{1}\left(C, x_{0} ; \kappa\right)$, defined in (21), in the limit $\kappa \rightarrow 0$, and are simply given by $\gamma_{0}\left(C, x_{0}\right)$ and $\gamma_{1}\left(C, x_{0}\right)$ in (16); viz.,

$$
\begin{gathered}
\gamma_{0}\left(C, x_{0}\right)=\operatorname{Hol}_{x_{0}}(A ; C), \\
\gamma_{1}\left(C, x_{0}\right)=\oint_{x \in\left(C, x_{0}\right)} \operatorname{Hol}_{x_{0}}^{x} B(x) \operatorname{Hol}_{x}^{x_{0}} .
\end{gathered}
$$

Under (27) and (28), they transform as

$$
\gamma_{i}\left(C, x_{0}\right) \rightarrow g\left(x_{0}\right) \gamma_{i}\left(C, x_{0}\right) g\left(x_{0}\right)^{-1}, \quad i=0,1,
$$

provided we fix $\psi\left(x_{0}\right)=0$, which can always be done. ${ }^{1}$

Thus, we are led to consider the v.e.v.s of $\operatorname{Tr}_{d} \gamma_{0}$ and $\operatorname{Tr}_{d} \gamma_{1}$. They, however, are rather trivial, for they correspond, respectively, to $J_{d}(C ; 0)=d$ and $(d / d h) J_{d}(C ; 0)=0$.

From a field-theoretical point of view, the reason of this triviality is the following: In perturbative $\mathrm{BF}$ theory (on $S^{3}$ ), nonvanishing v.e.v.s contain a number $n_{A}$ of fields $A$ not exceeding the number $n_{B}$ of fields $B .{ }^{1}$ Therefore, after expanding the holonomies in (29) in powers of $A$, one sees that only the zeroth-order term $\operatorname{Tr}_{(d-1) / 2} I=d$ (where $I$ is the group identity) survives in the v.e.v. of $\operatorname{Tr}_{(d-1) / 2} \gamma_{0}$; while, in the v.e.v. of $\operatorname{Tr}_{(d-1) / 2} \gamma_{1}$, only the first-order term does [the zerothorder term vanishing because it is the trace of an element of su(2)]. However, the latter v.e.v. (of the form $\left.\left\langle\oint_{C} A \oint_{C} B\right\rangle_{\mathrm{BF}, 0}\right)$ gives the self-linking number of the knot $C$ that, by the hypothesis of standard framing, is zero. (If one uses a more general framing, the self-linking number is the only information one gets from these v.e.v.s.)

This is the reason why in Ref. 1 one looked for composite observables that contain a higher number $n_{B}$ of fields $B$, and are invariant under (27) and (28). In particular, the generating function of the v.e.v.s of $\operatorname{Tr}_{(d-1) / 2}\left(\gamma_{1}\right)^{n}$ was taken into account, 


$$
W_{d}(C ; \lambda):=\sum_{n=0}^{\infty} \frac{\lambda^{n}}{n !}\left\langle\operatorname{Tr}_{(d-1) / 2} \gamma_{1}\left(C, x_{0}\right)^{n}\right\rangle_{\mathrm{BF}, 0}=\left\langle\operatorname{Tr}_{(d-1) / 2} \exp \left[\lambda \gamma_{1}\left(C, x_{0}\right)\right]\right\rangle_{\mathrm{BF}, 0},
$$

with $\lambda \in \mathrm{C}$. Again, as in (22), the rightmost term is only formal.

In Sec. V, we shall need (31) to study the limit $\kappa \rightarrow 0$ in (22), to which (31) is apparently related. However, there is a second, perhaps more natural, generating function to be considered, viz.,

$$
\widetilde{W}_{d}(C ; \lambda):=\left\langle\operatorname{Tr}_{(d-1) / 2} \exp \left[\lambda \beta\left(C, x_{0}\right)\right]\right\rangle_{\mathrm{BF}, 0},
$$

where

$$
\beta\left(C, x_{0}\right):=\gamma_{1}\left(C, x_{0}\right) \cdot \gamma_{0}\left(C, x_{0}\right)^{-1}=\oint_{C, x_{0}} \hat{B} \in \operatorname{su}(2) .
$$

Notice that the exponential in (32) is not formal, but it actually represents the exponential map from the algebra to the group.

The generating function $\widetilde{W}$ is particularly useful because it allows us to understand the meaning of the knot invariants related to higher-dimensional representations; indeed, by using (A7), one can easily prove that

$$
\begin{aligned}
& \widetilde{W}_{2 k}(C ; \lambda)=\sum_{l=1}^{k} \widetilde{W}_{2}(C ;(2 l-1) \lambda), \\
& \widetilde{W}_{2 k+1}(C ; \lambda)=1+\sum_{l=1}^{k} \widetilde{W}_{2}(C ; 2 l \lambda) .
\end{aligned}
$$

Then, a formal resummation of the geometric sums appearing in (34) and (35) gives

$$
\widetilde{W}_{d}(C ; \lambda)=\left\{\begin{array}{l}
\left\langle\operatorname{Tr}_{1 / 2} \frac{\exp \left[(d+1) \lambda \beta\left(C, x_{0}\right)\right]-1}{\exp \left[2 \lambda \beta\left(C, x_{0}\right)\right]-1}\right\rangle_{\mathrm{BF}, 0} \text { if } d \text { is even, } \\
\left\langle\operatorname{Tr}_{1 / 2} \frac{\exp \left[(d+1) \lambda \beta\left(C, x_{0}\right)\right]-1}{\exp \left[2 \lambda \beta\left(C, x_{0}\right)\right]-1}\right\rangle_{\mathrm{BF}, 0}+1 \quad \text { if } d \text { is odd. }
\end{array}\right.
$$

These formulas show that the limit

$$
\widetilde{W}(C ; \mu):=\lim _{d \rightarrow \infty, \lambda \rightarrow 0, d \lambda=\mu} \frac{\widetilde{W}_{d}(C ; \lambda)}{d}
$$

is well defined, and that

$$
\widetilde{W W}(C ; \mu)=\left\langle\operatorname{Tr}_{1 / 2} \frac{\exp \left[\mu \beta\left(C, x_{0}\right)\right]-1}{2 \mu \beta\left(C, x_{0}\right)}\right\rangle_{\mathrm{BF}, 0}=\sum_{n=0}^{\infty} \frac{1}{2(n+1) !}\left\langle\operatorname{Tr}_{1 / 2}\left[\mu \beta\left(C, x_{0}\right)\right]^{n}\right\rangle_{\mathrm{BF}, 0}
$$

Thus, by (32) and (38), we obtain

$$
\widetilde{W}_{2}(C ; \lambda)=2 \frac{d}{d \lambda}[\lambda \widetilde{W W}(C ; \lambda)]
$$

or equivalently, 


$$
\widetilde{W}(C ; \mu)=\frac{1}{\mu} \int_{0}^{\mu / 2} d x \widetilde{W}_{2}(C ; 2 x)
$$

Notice that (34) and (35) are actually discretizations of the Riemann integral in (40) with $x=l \lambda$.

A more rigorous proof of the above results can be obtained by expanding $\widetilde{W}_{2}$ in powers of $\lambda$ and using (34) and (35) to get the corresponding expansion of $\widetilde{W}_{d}$. Then one can use the asymptotic formula

$$
\sum_{l=1}^{k} l^{n} \stackrel{k \rightarrow \infty}{\sim} \frac{k^{n+1}}{n+1}
$$

to get the rightmost term in (38).

So far we have considered two different generating functions, viz., (31) and (32): in general, however, there are even more choices. Indeed, because of (30), a product of powers of $\gamma_{0}$ and $\gamma_{1}$ (in any order) is still an observable whose trace is invariant under (27) and (28).

To get rid of this arbitrariness in the definition of the generating function, we need the following.

Theorem 1: In pure BF theory, the knot invariant obtained by the v.e.v. of the trace of a function of $\gamma_{0}\left(C, x_{0}\right)$ and $\gamma_{1}\left(C, x_{0}\right)$ is equal to the knot invariant obtained by replacing $\gamma_{0}\left(C, x_{0}\right)$ with the identity of the group, provided the standard framing is chosen.

In Ref. 14, a proof of the theorem based on an explicit study of the Feynman integrals appearing in the evaluations of the v.e.v.s was given. Here, however, we give a simpler argument, based on the formal properties of the pure BF theory.

First of all we recall that, in the explicit evaluation of the v.e.v.s in BF theory, the choice of framing is done by evaluating all the holonomies in (29) on a companion knot $C^{\prime}$ obtained by $C$ in terms of a "small," nonvanishing normal displacement. ${ }^{1}$ Notice that the self-linking number of $C$ is then, by definition, the linking number of $C$ and $C^{\prime}$.

The proof of the theorem, then, essentially relies on the fact that, if this linking number vanishes (standard framing), one can deform $C^{\prime}$ in $\gamma_{0}$ in such a way that it can be completely unlinked from $C$ and, as such, shrunk to a point; so its holonomy, $\gamma_{0}$, becomes the identity.

This is possible since a knot $C^{\prime}$ appearing in $\gamma_{0}$ (not necessarily a framing for $C$ ) 'does not see itself." In fact, a small deformation of the knot $C^{\prime}$ at a certain point $x$ amounts to introducing curvature terms in $x$ (remember that $\gamma_{0}$ is a holonomy). However, in pure BF theory, the curvature vanishes everywhere but on the knot $C$, along which $\gamma_{1}$ is evaluated. (We refer to Ref. 1 for the proof that, in an observable, $A$ acts as a source for $d_{A} B$, while $B$ acts as a source for the curvature $F$.) Therefore, $C^{\prime}$ can be deformed freely as far as this deformation does not intersect $C$; selfintersections of $C^{\prime}$ are allowed, however.

When such a self-intersection occurs, one can split $C^{\prime}$ into two closed curves $C_{1}^{\prime}$ and $C_{2}^{\prime}$; correspondingly, the holonomy $\gamma_{0}\left(C^{\prime}\right)$ can be written as $\gamma_{0}\left(C_{1}^{\prime}\right) \cdot \gamma_{0}\left(C_{2}^{\prime}\right)$,

To prove the theorem, one just has to repeat this procedure until $C^{\prime}$ is replaced by a collection of circles $C_{1}^{\prime}$, all of which surround one single strand of $C$. Then one moves the $C_{1}^{\prime}$ s along $C$, and cuts and splices them together again to form a circle $C^{\prime \prime}$.

Since all these deformations do not affect the linking between $C$ and $C^{\prime}$, the linking number of $C$ and $C^{\prime \prime}$ is the same as the linking number of $C$ and $C^{\prime}$. If this linking number vanishes (standard framing), then the circle $C^{\prime \prime}$ and the knot $C$ are unlinked, and $C^{\prime \prime}$ can be shrunk to a point. This concludes the proof of Theorem 1.

As a consequence of Theorem 1 , we have the following.

Corollary 1: $W_{d}(C ; \lambda)$ is the most general v.e.v. one can consider in pure BF theory if the standard framing is chosen. 
Moreover, we can freely switch from $W_{d}$ in (31) and $\widetilde{W}_{d}$ in (32). Thus, by (34) and (35), the higher-representation knot invariants $W_{d}$ can be related to the fundamental-representation knot invariant $W_{2}$; besides, the limit

$$
W W(C ; \mu):=\lim _{d \rightarrow \infty, \lambda \rightarrow 0, d \lambda=\mu} \frac{W_{d}(C ; \lambda)}{d},
$$

is well defined and, by (39),

$$
W_{2}(C ; \lambda)=2 \frac{d}{d \lambda}[\lambda W W(C ; \lambda)]
$$

We conclude this section with an important remark. Pure BF theory is known to be exact in saddle-point approximation ${ }^{16}$ as far as the partition function is concerned. This result is a simple consequence of the fact that one can arbitrarily change the "Planck constant" in front of the action by simply rescaling the field $B$. Since the partition function (or the v.e.v. of an observable not containing $B$ ) is not affected by this rescaling, one can send the Planck constant to zero.

This, of course, cannot be done when one computes the v.e.v. of an observable containing $B$, as in (31) or (32). In this case one sees that the parameter $\lambda$ actually plays the role of the Planck constant, and, of course, $W_{d}$ is not independent of $\lambda$.

Notice, however, that $W W$ corresponds to the limit $\lambda \rightarrow 0$, so it should be possible to compute it by using the saddle-point approximation. Then, by (42), it is possible to recover $W_{2}$-and hence, by (34) and (35), all the $W_{d}$ 's-from $W W$.

Thus, even if pure BF theory with $B$-dependent observables is not exact in saddle-point approximation, the saddle-point approximation turns out to be all that one needs.

\section{TURNING OFF THE COSMOLOGICAL CONSTANT}

In this section we want to show that, in the limit of vanishing cosmological constant, the BF-theory generating function (22) is related to both the pure-BF-theory generating function (31) and the Melvin-Morton function (4).

To establish the former relation, we first observe that, in order for the significant observable $\gamma_{1}$ to survive in the limit $\kappa \rightarrow 0$, we have to send $|x| \rightarrow \infty$ at the same time with the prescription that $x \kappa=\lambda$ be finite. If we work with the standard framing, by Theorem 1 the observable $\gamma_{0}$ can be replaced by the identity of the group; so (22) diverges as $e^{x}$; thus, we are led to consider

$$
\widetilde{E}(C ; x, h):=e^{-x} E(C ; x, h) .
$$

By using the exponential representation of (22), we can write

$$
\widetilde{E}(C ; x, h)=\left\langle\operatorname{Tr}_{1 / 2} \exp \left\{x\left[\kappa \gamma_{1}\left(C, x_{0}\right)+O\left(\kappa^{2}\right)\right]\right\}\right\rangle_{\mathrm{BF}, \kappa}
$$

and get

$$
\lim _{|x| \rightarrow \infty, h \rightarrow 0, x h=\hbar} \widetilde{E}(C ; x, h)=W_{2}(C ; \lambda), \quad \hbar=4 \pi i \lambda .
$$

In Appendix C, we give a more careful proof of (44).

Notice that the limit in (44) holds irrespectively of how $x$ is sent to infinity in the complex plane. Therefore, if one sees $\widetilde{E}$ as a meromorphic function of $x$ and $\hbar$, this implies that the regular part in $x$ of $\widetilde{E}$ vanishes. Thus, $\widetilde{E}$, now rewritten in terms of $x$ and $h$, does not contain more powers of $x$ than of $h$. In other words, if $\widetilde{E}$ is expanded as a rational power series, 


$$
\widetilde{E}(C ; x, h)=\sum_{l, m=0}^{\infty} \epsilon_{l m}(C) x^{l} h^{m}
$$

then $\epsilon$. is an upper triangular matrix,

$$
\epsilon_{j m}(C)=0, \text { if } j>m
$$

The relation between the BF-theory generating function and the Melvin-Morton function is obtained by exploiting (25) (see Appendix D for details). We arrive to the following conclusions:

$$
\epsilon . \text { uppper triangular } \Leftrightarrow b . \text { upper triangular, }
$$

and

$$
\epsilon_{n n}(C)=2(1+n) b_{n n}(C)
$$

where $\epsilon$. and $b .$. are defined, respectively, in (45) and (1). Notice that (47) and (46) give a new proof of (3).

Since $E$ is related to $W_{2}$ and to $J J$ in the same limit $\kappa \rightarrow 0$, we deduce that a relation exists between $W_{2}$ and $J J$. Actually, by (1), (44), (45), and (48), we obtain

$$
W_{2}(C ; \lambda)=2 \frac{d}{d \hbar}[\hbar J J(C ; \hbar)], \quad \hbar=4 \pi i \lambda
$$

In order to recognize the knot invariant given by $W_{2}$, we can now resort to (5) and obtain

$$
W_{2}(C ; \lambda)=2\left[1+\left(\frac{z}{2}\right)^{2}\right]^{1 / 2} \frac{d}{d z} \frac{z}{\Delta(C ; z)}, \quad z=2 i \sin (2 \pi \lambda),
$$

and, in particular,

$$
W_{2}(O ; \lambda)=2\left[1+\left(\frac{z}{2}\right)^{2}\right]^{1 / 2}, \quad z=2 i \sin (2 \pi \lambda),
$$

where $O$ is the unknot and we have chosen the normalization $\Delta(O ; z)=1$ (cfr. Sec. II). Thus, the normalized knot invariant

$$
\langle C\rangle_{2}(\lambda):=\frac{W_{2}(C ; \lambda)}{W_{2}(O ; \lambda)}
$$

defined in Ref. 1, satisfies

$$
\langle C\rangle_{2}(\lambda)=\frac{d}{d z} \frac{z}{\Delta(C ; z)}, \quad z=2 i \sin (2 \pi \lambda)
$$

In conclusion, we have shown that $W_{2}$ is related to the first derivative of the inverse of the Alexander-Conway polynomial. By (34) and (35), $W_{d}$ is given by a finite sum of $W_{2}$ s, evaluated at different $\lambda$ s. Concerning the limit $d \rightarrow \infty$, we see that (42) and (49), together with the property $W W(C ; 0)=J J(C ; 0)=1$, imply

$$
W W(C ; \lambda)=J I(C ; \hbar), \quad \hbar=4 \pi i \lambda,
$$

or, because of (5), 


$$
4 \pi i \lambda W W(C ; \lambda)=\frac{z}{\Delta(C ; z)}, \quad z=2 i \sin (2 \pi \lambda) .
$$

The above results, together with Corollary 1 , prove the following.

Theorem 2: The set of the unframed knot invariants that can be obtained from the SU(2)-BF theory coincides with the set of the coefficients of (the inverse of) the Alexander-Conway polynomial.

In Ref. 1, the authors conjectured a relation between the Alexander-Conway polynomials and the pure BF theory based on second-order calculations in the perturbative expansion. Theorem 2 supersedes this conjecture and provides the correct relation.

\section{THE PERTURBATIVE EXPANSION OF $w_{d}$}

In this section we want to compare the results we have proved in Sec. $V$ with those obtained in Ref. 1 in the framework of "perturbative" BF theory.

By "perturbative" evaluation of (31), one means an expansion of $W_{d}(C ; \lambda)$ (or of its generalization $W_{G, K}(C ; \lambda)$, where $G$ is a compact group and $R$ a representation) in powers of $\lambda$,

$$
W_{G, R}(C ; \lambda)=\sum_{n=0}^{\infty} w_{n}(G, R ; C) \lambda^{n}
$$

where the knot invariants $w_{n}(G, R ; C)$ (actually, they are Vassiliev invariants ${ }^{17}$ ) are computed in terms of Feynman integrals.

The first property of the expansion (56) shown in Ref. 1 is that, owing to a symmetry of the corresponding Feynman integrals, odd-order terms vanish,

$$
w_{2 n+1}(G, R ; C)=0 \text {. }
$$

Thus, $W$ is an even function:

$$
W_{G, R}(C ;-\lambda)=W_{G, R}(C ; \lambda) .
$$

In the case $G=\mathrm{SU}(2)$, by using (50), (34), and (35), we see that (58) is in accordance with the fact that the Alexander-Conway polynomial $\Delta(C ; z)$-for a single knot $C$-is an even function of $z$.

A further computation done in Ref. 1, for the case $G=\mathrm{SU}(N)$, showed that, up to the second order, (56) reads

$$
W_{G, R}(C ; \lambda)=\operatorname{dim} R\left[1+(4 \pi \lambda)^{2} c_{2}(R) c_{v} \rho(C)+O\left(\lambda^{4}\right)\right]
$$

where

(i) $\operatorname{dim} R$ and $c_{2}(R)$ are, respectively, the dimension and the quadratic Casimir of the representation $R$;

(ii) $\quad c_{v}=N$ is the quadratic Casimir of the adjoint representation; and

(iii) $\rho(C)$ is the knot invariant studied in Refs. 18 and 19 in the framework of the ChernSimons theory.

In Refs. 18 and $19, \rho(C)$ was proved to be related to the second coefficient of the AlexanderConway polynomial; viz., if one writes

$$
\Delta(C ; z)=\sum_{n=0}^{\infty} a_{n}(C) z^{n}, \quad a_{0}(C)=1, \quad a_{1}(C)=0
$$

(where only a finite number of coefficients are nonvanishing), then 


$$
\rho(C)=2 a_{2}(C)+\rho(\bigcirc)
$$

Moreover, a direct computation ${ }^{18}$ shows that

$$
\rho(O)=-\frac{1}{12}
$$

Thus, one can write (59) as

$$
W_{G, R}(C ; \lambda)=\operatorname{dim} R\left[1+(4 \pi \lambda)^{2} 2 c_{2}(R) c_{v}\left(a_{2}(C)-\frac{1}{24}\right)+O\left(\lambda^{4}\right)\right]
$$

If we now expand (50) as

$$
\begin{gathered}
W_{2}(C ; \lambda)=2\left[1+\frac{1}{8} z^{2}+O\left(z^{4}\right)\right]\left[1-3 a_{2}(C) z^{2}+O\left(z^{4}\right)\right]=2\left[1+3 z^{2}\left(\frac{1}{24}-a_{2}(C)\right)+O\left(z^{4}\right)\right] \\
z=4 \pi i \lambda+O\left(\lambda^{2}\right)
\end{gathered}
$$

we get a complete agreement with (63) in the case where $G=\mathrm{SU}(2)$ and the fundamental representation, $R_{2}$, is chosen; for, in this case, $c_{v}=2$ and $c_{2}\left(R_{2}\right)=\frac{3}{4}$.

We can also compare the second-order expansion of (34) and (35) with (63) in the case where $G=\mathrm{SU}(2)$ and $R_{d}$ is the irreducible representation of dimension $d$. This is done by noticing that one can compute the sums appearing in (34) and (35) as

$$
\sum_{l=1}^{k}(2 l-1)^{2}=\frac{1}{3} k\left(4 k^{2}-1\right), \quad \sum_{l=1}^{k} l^{2}=\frac{2}{3} k(k+1)(2 k+1) .
$$

Thus, one achieves complete agreement with (63) since

$$
\begin{gathered}
c_{2}\left(R_{2 k}\right)=\frac{(2 k-1)(2 k+1)}{4}, \\
c_{2}\left(R_{2 k+1}\right)=k(k+1) .
\end{gathered}
$$

By using (34) and (35), it is possible to see that-in agreement with the perturbative result of Ref. 1 -the coefficients $w_{n}$ in (56) are given by the product of a function depending only on the representation and a function depending only on the knot, viz.,

$$
w_{2 n}\left(\mathrm{SU}(2), R_{d} ; C\right)=d g_{2 n}(d) v_{2 n}(C) \text {. }
$$

If we normalize $g_{2 n}(2)=\frac{1}{2}$, then, by (34) and (35), we get an explicit formula for the $g \mathrm{~s}$ :

$$
\begin{gathered}
g_{2 n}(2 k)=\frac{1}{2 k} \sum_{l=1}^{k}(2 l-1)^{2 n}, \\
g_{2 n}(2 k+1)=\frac{1}{2 k+1} \sum_{l=1}^{k}(2 l)^{2 n} .
\end{gathered}
$$

By (64) and (65), we can also express the $g s$ in terms of the quadratic Casimirs: 


$$
\begin{gathered}
g_{0}=\frac{1}{2}, \quad g_{2}=\frac{1}{3} c_{2} c_{v}, \\
g_{4}=\frac{1}{15}\left[6\left(c_{2} c_{v}\right)^{2}-c_{2} c_{v}^{3}\right], \quad g_{6}=\frac{1}{21}\left[12\left(c_{2} c_{v}\right)^{3}-6 c_{2}^{2} c_{v}^{4}+c_{2} c_{v}^{5}\right],
\end{gathered}
$$

In general, the highest power of $c_{2}$ in $g_{2 n}$ is $n$.

In Ref. 1, it was conjectured that the only group factor to appear in $g_{2 n}$ was $\left(c_{2} c_{v}\right)^{n}$. From this conjecture, together with some field-theoretical arguments, it was concluded that, for some $t$,

$$
\frac{W_{G, R}(C, \lambda)}{W_{G, R}(O, \lambda)}=[\Delta(C ; z)]^{t}, \quad z \propto \sqrt{c_{2}(R) c_{v}} \lambda+O\left(\lambda^{3}\right)
$$

By (69), we see that the conjecture is wrong; by (49), we see that so is the conclusion (70).

It is, however, interesting to show that, if we retain only the terms $\left(c_{2} c_{v}\right)^{n}$ in (69), then the corresponding knot invariant is actually the $(-1)$-power of the Alexander-Conway polynomial. More precisely, we define the "truncated" cocfficicnts

$$
g_{2 n}^{(\mathrm{tr})}(d)=\left[c_{2}\left(R_{d}\right) c_{v}\right]^{n} \gamma_{2 n}
$$

as the coefficients obtained by neglecting lower powers of $c_{2}\left(R_{d}\right)$ in (69), and the "truncated" knot invariant as

$$
W_{d}^{(\mathrm{rr})}(C ; \lambda):=\sum_{n=0}^{\infty} d g_{2 n}^{(\mathrm{tr})}(d) v_{2 n}(C) \lambda^{2 n}
$$

Since $c_{v}=2$ and $c_{2}\left(R_{d}\right) \sim d^{2} / 4$ as $d \rightarrow \infty$, the truncated coefficients $g_{2 n}^{(\text {tr })}(d)$ are the leading terms of the true coefficients $g_{2 n}(d)$; thus, by (41) and (71),

$$
W W(C ; \lambda)=\sum_{n=0}^{\infty} \frac{\gamma_{2 n}}{2^{n}} v_{2 n}(C) \lambda^{2 n}
$$

By comparing (73) with (72), we eventually get

$$
W_{d}^{(\mathrm{tr})}(C ; \lambda)=d W W\left(C ; \sqrt{2 c_{2}\left(R_{d}\right) c_{v}} \lambda\right) ;
$$

thus, by (55), we see that

$$
\frac{W_{d}^{(\mathrm{tr})}(C ; \lambda)}{W_{d}^{(\mathrm{tr})}(O ; \lambda)}=\frac{1}{\Delta(C ; z)}, \quad z=2 i \sin \left(2 \pi \sqrt{2 c_{2}\left(R_{d}\right) c_{v}} \lambda\right)
$$

Thus, if the truncated knot invariants are used, (70) holds.'

\section{CONCLUSIONS}

In this paper we have discussed the unframed knot invariants coming from BF theories. Even if most of our results hold only for SU(2), we point out that Theorem 1 and Corollary 1, as well as the computation in Appendix $C$ (with a slight abuse of notation), hold in general.

It would be interesting to generalize some of the other results to different groups and to consider links as well. However, the present case, i.e., knot observables in the theory based on $\mathrm{SU}(2)$, seems to be interesting enough to deserve further investigation. 
Indeed, as we have noticed at the end of Sec. IV, it turns out that a saddle-point computation is enough to completely describe the pure BF theory. We defer to a forthcoming paper the related functional-integral computation.

Notice that this property of pure BF theory sets it at the boundary between TQFTs of Witten's and Schwarz's type: The former are twisted supersymmetric gauge theories (see, e.g., Ref. 20), whose main property - an effect of the twisted supersymmetry-is their independence of both the metric and the coupling constant, which makes them topological as well as exact in saddle-point approximation. The latter are topological gauge theories, as the Chern-Simons or the BF theories, whose dependence on the coupling constant is unavoidable. The pure BF theory formally belongs to the latter type, but, as the theories of the former type, is completely determined by its weak-coupling limit.

Moreover, as we have proved in Sec. V, the pure BF theory corresponds to the first diagonal in the $(h, d)$ expansion of the colored Jones function. A description of the upper diagonals is still missing (see Ref. 21 for a first attempt); so it is natural to look for generalizations of the pure BF theory, i.e., for further variations of the Chern-Simons theory, that could correspond to these upper diagonals and, possibly, give them a better understanding (see Ref. 13 for a different approach). We are investigating along these lines.

\section{ACKNOWLEDGMENTS}

I thank A. Jaffe, T. Kerler, A. Leśniewski, M. Martellini, and M. Rinaldi for helpful conversations. I am especially thankful to P. Cotta-Ramusino for a number of very useful discussions and for constant advice.

This work was supported by INFN Grant No. $5077 / 94$.

\section{APPENDIX A: SOME USEFUL IDENTITIES FOR THE CHARACTERS OF SU(2)}

In this section we recall some properties of the group $\mathrm{SU}(2)$ that are necessary for this paper. In particular, we are interested in an identity relating $\operatorname{Tr}_{1 / 2} g^{n}$ to the traces of $g$ in other representations, or, in other words, we want to give $\operatorname{Tr}_{1 / 2} g^{n}$ an expression in primitive characters,

$$
\operatorname{Tr}_{1 / 2} g^{n}=\sum_{k=0}^{\infty} \phi_{n k} \chi_{k / 2}(g)
$$

where

$$
\chi_{s}(g):=\operatorname{Tr}_{s} g, \quad 2 s \in \mathbf{Z} .
$$

Owing to the Peter-Weil Theorem, this can be done since $\operatorname{Tr}_{1 / 2} g^{n}$ depends only on the conjugacy class of $g$ and belongs to $L^{2}(\mathrm{SU}(2), \mathrm{C})$. By a conjugacy transformation, one can always write $g$ as

$$
g=h e^{i \alpha R_{3}} h^{\dagger},
$$

where $R_{3}$ is the (Hermitean) generator of the Cartan subalgebra. Noticing that the spectrum of $R_{3}$ in the representation of spin $l$ is given by $\{-2 l,-2(l-1), \ldots, 2(l-1), 2 l\}$, one obtains

$$
\operatorname{Tr}_{1 / 2} g^{n}=e^{i n \alpha}+e^{-i n \alpha},
$$

and

$$
\operatorname{Tr}_{1} g=e^{2 i l \alpha}+e^{2 i(l-1) \alpha}+\cdots+e^{-2 i l \alpha}=\sum_{m=-l}^{l} e^{2 i m \alpha},
$$


where the last sum is meant to be over integers or half-integers according to the fact that $l$ is integer or half-integer. By (A4) and (A5), it immediately follows that, for $n \geqslant 2$,

$$
\operatorname{Tr}_{1 / 2} g^{n}=\operatorname{Tr}_{n / 2} g-\operatorname{Tr}_{n / 2-1} g, \quad n \in \mathbf{Z}
$$

By induction one can also prove that

$$
\operatorname{Tr}_{s} g= \begin{cases}\sum_{l=1}^{s+1 / 2} \operatorname{Tr}_{1 / 2} g^{2 l-1} & \text { if } s \text { is half-integer, } \\ 1+\sum_{l-l}^{s} \operatorname{Tr}_{1 / 2} g^{2 l} & \text { if } s \text { is integer. }\end{cases}
$$

Moreover, by (A7) and by the fact that

$$
\operatorname{Tr}_{1 / 2} \exp (i \mathbf{a} \cdot \mathbf{R})=2 \cos a,
$$

with $\mathbf{a} \in \mathbb{R}^{3}$ and $\mathbf{R}=\left(R^{1}, R^{2}, R^{3}\right)$ the Pauli matrices, one also obtains

$$
\operatorname{Tr}_{s} \exp (i \mathbf{a} \cdot \mathbf{R})=\frac{\sin (d a)}{\sin (a)}
$$

where $d=2 s+1$ is the dimension of the representation of spin $s$.

Since, for a given $g, \operatorname{Tr}_{n / 2} \delta$ is a map defined on the positive integers, its analytic continuation over the whole complex plane is uniquely defined and is actually given by (A8).

\section{APPENDIX B: PROPERTIES OF THE GENERATING FUNCTION FOR COLORED JONES FUNCTIONS}

In this section we explore the properties of the generating function for colored Jones functions defined in (6). By (1), we can write

$$
f(C ; x, h)=\sum_{d=1}^{\infty} \frac{x^{d}}{d !} d \sum_{j, m=0}^{\infty} b_{j m}(C)(d-1)^{j} h^{m}=x \sum_{j, m=0}^{\infty} B_{j}(x) b_{j m}(C) h^{m},
$$

where

$$
B_{j}(x)=\sum_{d=0}^{\infty} \frac{x^{d}}{d !} d^{j}=\left.\left(\frac{\partial^{j}}{\partial \alpha^{j}}\right)\right|_{\alpha=\log x} \sum_{d=0}^{\infty} \frac{e^{\alpha d}}{d !}=\left.\left(\frac{\partial^{j}}{\partial \alpha^{j}}\right)\right|_{\alpha=\log x} \exp \left(e^{\alpha}\right) .
$$

By repeatedly applying Leibniz's rule, we obtain

$$
B_{j}(x)=e^{x} P_{j}(x)=e^{x}\left[x^{j}+O\left(x^{j-1}\right)\right],
$$

where $P_{j}(x)$ is a polynomial of degree $j$ starting with $x^{j}$, viz.,

$$
P_{j}(x)=\sum_{l=0}^{j} x^{l} c_{l j}, \quad c_{j j}=1
$$

Notice that (B4) implies that $c .$. is an upper triangular matrix:

$$
c_{l j}(C)=0, \text { if } l>j
$$

The coefficients $c_{l j}$ can easily be computed if we consider the following generating function, 


$$
B(\rho, x):=\sum_{j=0}^{\infty} \frac{p^{j}}{j !} B_{j}(x)
$$

which, by (B2), can be written as

$$
B(\rho, x)=\left.\exp e^{(\alpha+\rho)}\right|_{\alpha=\log x}=e^{x} \exp \left[x\left(e^{\rho}-1\right)\right] .
$$

Thus, by (B3), we obtain

$$
P(\rho, x):=\sum_{j=0}^{\infty} \frac{\rho^{j}}{j !} P_{j}(x)=\sum_{n=0}^{\infty} \frac{x^{n}}{n !}\left(e^{p}-1\right)^{n},
$$

while, by (B4), we have

$$
P(\rho, x)=\sum_{n, j=0}^{\infty} x^{n} c_{n j} \frac{\rho^{j}}{j !} .
$$

By comparing the two different expansions of $P$ in powers of $\rho$ given by (B8) and (B9), we obtain eventually

$$
c_{n j}=\frac{(-1)^{n}}{n !} \sum_{l=0}^{n}\left(\begin{array}{l}
n \\
l
\end{array}\right)(-1)^{l} l^{j}
$$

where, by convention, $0^{0}=1$. write

We want now to compute the coefficients $\tilde{b} .$. defined in (8). By (7), (B1), and (B3), we can

$$
\widetilde{f}(C ; x, h)=\sum_{j, m=0}^{\infty} P_{j}(x) b_{j m}(C) h^{m}
$$

so by $(8)$,

$$
\tilde{b}_{n m}(C)=\sum_{j=0}^{\infty} c_{n j} b_{j m}(C)
$$

Since the matrices $\widetilde{b} .$. and $b$. are related by the matrix $c$.. that, by (B5), is upper triangular, we conclude that (9) holds. Moreover, if one knows that either $\widetilde{b}$.. or $b .$. is upper triangular-and by (3) we know that this is true for the latter-then one has

$$
\widetilde{b}_{m m}(C)=b_{m m}(C)
$$

This implies that, in the limit considered in (11), we can write

$$
\widetilde{f}(C ; x, h)=\sum_{m=0}^{\infty} \widetilde{b}_{m m}(C) \hbar^{m}+O\left(\frac{\hbar}{x}\right)=\sum_{m=0}^{\infty} b_{m m}(C) \hbar^{m}+O\left(\frac{\hbar}{x}\right)
$$

so by (4), (11) holds. In order to prove (12), we only have to notice that the operator $(x \partial / \partial x)^{n}$, when applied to $x^{j}$ simply produces a factor $j^{n}$; so

$$
\left(x \frac{\partial}{\partial x}\right)^{n} \widetilde{f}(C ; x, h)=\sum_{m=0}^{\infty} m^{n} \widetilde{b}_{m m}(C) \hbar^{m}+O\left(\frac{\hbar}{x}\right)=\sum_{m=0}^{\infty} m^{n} b_{m m}(C) \hbar^{m}+O\left(\frac{\hbar}{x}\right) .
$$


By comparison with the effect of the operator $(\hbar d / d \hbar)^{n}$ on (4), we obtain (12).

\section{APPENDIX C: FROM E TO $W_{2}$ AS $\kappa \rightarrow 0$}

In this section we want to clarify the limit in (44). By (43), (22), and (14), we have

$$
\widetilde{E}(C ; x, h)=e^{-x} \sum_{n=0}^{\infty} \frac{x^{n}}{n !}\left\langle\operatorname{Tr}_{1 / 2}\left[\gamma_{0}\left(C, x_{0}\right)+\kappa \gamma_{1}\left(C, x_{0}\right)+O\left(\kappa^{2}\right)\right]^{n}\right\rangle_{\mathrm{BF}, \kappa},
$$

where $h=4 \pi i \kappa$.

In Refs. 3 and 14 , it is shown that, in an observable, the field $B$ represents a source for $F+\kappa^{2} B \wedge B$, while the field $A$ is a source only for $d_{A} B$. Since a variation in the framing (i.e., the companion knot along which we integrate the field $A$ ) is still given by inserting a curvature term, we can repeat the steps of the proof of Theorem 1 and show that, if the standard framing is chosen, $\Gamma_{0}$ can be replaced by $I+O\left(\kappa^{2}\right)$, where $I$ is the group identity. As a consequence, $(\mathrm{Cl})$ now reads

$$
\widetilde{E}(C ; x, h)=e^{-x} \sum_{n=0}^{\infty} \frac{x^{n}}{n !}\left\langle\operatorname{Tr}_{1 / 2}\left[I+\kappa \gamma_{1}\left(C, x_{0}\right)+O\left(\kappa^{2}\right)\right]^{n}\right\rangle_{\mathrm{BF}, \kappa}
$$

By using Newton's binomial formula, one can easily prove that (C2) can also be written as

$$
\widetilde{E}(C ; x, h)=\sum_{n=0}^{\infty} \frac{x^{n}}{n !}\left\langle\operatorname{Tr}_{1 / 2}\left[\kappa \gamma_{1}\left(C, x_{0}\right)-O\left(\kappa^{2}\right)\right]^{n}\right\rangle_{\mathrm{BF}, \kappa}
$$

or, setting $\lambda=x \kappa$,

$$
\widetilde{E}(C ; x, h)=\sum_{n=0}^{\infty} \frac{\lambda^{n}}{n !}\left\langle\operatorname{Tr}_{1 / 2}\left[\gamma_{\mathrm{l}}\left(C, x_{0}\right)+O(\kappa)\right]^{n}\right\rangle_{\mathrm{BF}, \kappa}
$$

Now, sending $\kappa \rightarrow 0$, with $\lambda$ fixed, gives (44).

\section{APPENDIX D: FROM E TO $J J$ AS $h \rightarrow 0$}

In this section we consider the relation between the BF-theory generating function $E$ and the Melvin-Morton function as the expansion parameter $h$ is sent to zero.

The starting point is relation (25), which, by (7), can be rewritten in terms of $\widetilde{f}$. Actually, the second term on the rhs of $(25)$ is easily seen to be

$$
\partial_{x} f(C ; x, h)=e^{x}(x+1) \widetilde{f}(C ; x, h)+e^{x} x \partial_{x} \widetilde{f}(C ; x, h),
$$

while the computation of the last term requires more work. First of all, it is useful to introducc the following notation:

$$
I_{n}[g](x)=\int_{0}^{x} d \xi e^{\xi} g^{(n)}(\xi)
$$

where $g$ is a generic analytic function and $g^{(n)}$ its $n$th derivative. By integrating by parts, one can prove the recursion rule

$$
I_{n}[g](x)=e^{x} g^{(n)}(x)-g^{(n)}(0)-I_{n+1}[g](x),
$$

which implies that 


$$
I_{0}[g](x)=e^{x} \sum_{n=0}^{\infty}(-1)^{n} g^{(n)}(x)-\sum_{n=0}^{\infty}(-1)^{n} g^{(n)}(0)
$$

Now we set

$$
g(x)=x \widetilde{f}(C ; x, h)
$$

(where $C$ and $h$ are supposed to be fixed), and note that, accordingly,

$$
g^{(n)}(x)=n \partial_{x}^{n-1} \widetilde{f}(C ; x, h)+x \partial_{x}^{n} \widetilde{f}(C ; x, h)
$$

Then we use (D4) to compute the last term in (25) as

$$
\int_{0}^{x} d \xi f(C ; \xi, h)=e^{x}\left[(x-1) \widetilde{f}(C ; x, h)-x \partial_{x} \widetilde{f}(C ; x, h)+R(C ; x, h)\right]+c(C ; h),
$$

where

$$
R(C ; x, h)=\sum_{n=2}^{\infty}(-1)^{n}\left[n \partial_{x}^{n-1} \widetilde{f}(C ; x, h)+x \partial_{x}^{n} \widetilde{f}(C ; x, h)\right]
$$

and

$$
c(C ; h)=\sum_{n=0}^{\infty}(-1)^{n}(n+1) \partial_{x}^{n} \widetilde{f}(C ; 0, h)
$$

Therefore, by (43), (25), (D1), and (D7), we obtain

$$
\widetilde{E}(C ; x, h)=2\left[\widetilde{f}(C ; x, h)+x \partial_{x} \widetilde{f}(C ; x, h)\right]+R(C ; x, h)+e^{-x}[1-c(C ; h)] .
$$

We defer to the end of this section the proof that $c(C ; h)=1$, cf. (D23); as a consequence of this fact, (D10) actually reads

$$
\widetilde{E}(C ; x, h)=2\left[\widetilde{f}(C ; x, h)+x \partial_{x} \widetilde{f}(C ; x, h)\right]+R(C ; x, h) .
$$

Now we want to reexpress (D11) as a relation between the coefficients of the power series expansions of $\widetilde{E}$ and $\widetilde{f}$. Indeed, by (8), the terms in square brackets in (D11) can be written as

$$
\sum_{n, l=0}^{\infty}(1+l) \tilde{b}_{l m}(C) x^{l} h^{m}
$$

while

$$
R(C ; x, h)=\sum_{n=2}^{\infty} \sum_{l, m=0}^{\infty}(-1)^{n} \frac{(n+l) !}{l !} \widetilde{b}_{l+n-1, m}(C) x^{l} h^{m}
$$

Therefore, by (45), we get eventually

$$
\epsilon_{l m}(C)=\sum_{j=0}^{\infty} n_{l j} \widetilde{b}_{j m}(C)
$$

with 


$$
n_{l j}=2(1-l) \delta_{l j}+\sum_{n=2}^{\infty}(-1)^{n} \frac{(n+l) !}{l !} \delta_{l+n-1, j}
$$

( $\delta$. being the Kronecker delta). Since $\eta$. is an upper triangular matrix, by (D13) we have that

$$
\epsilon . \text { upper triangular } \Leftrightarrow \widetilde{b .} \text {. upper triangular. }
$$

Finally, by (9), we conclude that (47) holds. Moreover, (D14) and (B13) imply (48).

We conclude this section by showing that the function $c(C ; h)$, defined in (D9), is a constant equal to one.

We start by considering the expansion of $c$ in powers of $h$. By (8), we obtain

$$
c(C ; h)=\sum_{n, m=0}^{\infty}(-1)^{n}(n+1) ! \widetilde{b}_{n m}(C) h^{m}=\sum_{j, m=0}^{\infty} D_{j} b_{j m}(C) h^{m}
$$

where, by (B12),

$$
D_{j}=\sum_{n=0}^{\infty}(-1)^{n}(n+1) ! c_{n j}
$$

Notice that, by (B5), (D17) is actually a finite sum. By (B10), we can also write

$$
D_{j}=\sum_{n=0}^{\infty} \sum_{l=0}^{n}(n+1)\left(\begin{array}{l}
n \\
l
\end{array}\right)(-1)^{l} l^{j}
$$

Let us consider now the generating function

$$
D(p):=\sum_{j=0}^{\infty} \frac{p^{j}}{j !} D_{j}
$$

By (D18), it follow's that

$$
D(\rho)=\sum_{n=0}^{\infty}(n+1) \sum_{l=0}^{n}\left(\begin{array}{l}
n \\
l
\end{array}\right)(-1)^{l} e^{\rho l}=\sum_{n=0}^{\infty}(n+1)\left(1-\epsilon^{\rho}\right)^{n}=e^{-2 \rho} .
$$

By comparing the expansion of $D$ in powers of $\rho$ in (D19) with the expansion of $e^{-2 \rho}$, we conclude that

$$
D_{j}=(-2)^{j}
$$

Thus, (D16) reads

$$
c(C ; h)=\sum_{j, m=0}^{\infty} b_{j m}(C)(-2)^{j} h^{m}=-J_{-1}(C ; h),
$$

where the last identity follows from (1). However, by (2), we have

$$
J_{-1}(C ; h)=-J_{1}(C ; h)=-1 \text {. }
$$

Therefore, we conclude that

$$
c(C ; h)=1
$$


'A. S. Cattaneo, P. Cotta-Ramusino, and M. Martellini, "Three-dimensional BF theories and the Alexander-Conway invariant of knots," Nucl. Phys. B 436, 355-382 (1995).

2A. S. Schwarz, "The partition function of degenerate quadratic functionals and Ray-Singer invariants," Lett. Math. Phys. 2, 247-252 (1978).

${ }^{3}$ A. S. Cattaneo, P. Cotta-Ramusino, J. Fröhlich, and M. Martellini, "Topological BF theories in three and four dimensions," J. Math. Phys. 36, 6137-6160 (1995).

${ }^{4}$ E. Witten, " $(2+1)$-dimensional gravity as an exactly soluble system," Nucl. Phys. B 311, 46-78 (1988).

'A. Ashtekar, C. Rovelli, and L. Smolin, "Weaving a classical geometry with quantum threads," Phys. Rev. Lett. 69, 237-240 (1992).

${ }^{6}$ A. S. Cattaneo, P. Cotta-Ramusino, A. Gamba, and M. Martellini, “The Donaldson-Witten invariants in pure 4D-QCD with order and disorder 't Hooft-like operators," Phys. Lett. B 355, 245-254 (1995).

${ }^{7} \mathrm{D}$. Anselmi, "On field theory quantization around instantons," hep-th/9507167; "Topological field theory and confinement," hep-th/9504049.

${ }^{8} \mathrm{~J}$. Fröhlich, R. Götschmann, and P. A. Marchetti, "Bosonization of fermi systems in arbitrary dimensions in terms of gauge forms," J. Phys. A 28, 1169-1204 (1995).

${ }^{9}$ E. Witten, "Quantum field theory and the Jones polynomial," Commun. Math. Phys. 121, 351-399 (1989).

${ }^{10}$ P. M. Melvin and H. R. Morton, "The coloured Jones function," Commun. Math. Phys. 169, 501-520 (1995).

"D. Bar-Natan and S. Garoufalidis, "On the Melvin-Morton-Rozansky conjecture," Harvard University preprint, July 1994 (available at ftp://ftp.ma.huji.ac.il/drorbn).

${ }^{12}$ L. Rozansky, "A contribution of the trivial connection to Jones polynomial and Witten's invariants of $3 \mathrm{~d}$ manifolds. I," Commun. Math. Phys. 175, 275-296 (1996); "A contribution of the trivial connection to Jones polynomial and Witten's invariants of 3d manifolds. II," Commun. Math. Phys. 175, 297-318 (1996).

${ }^{13}$ A. Kricker, B. Spence, and I. Aitchison, "Cabling the Vassiliev Invariants," q-alg/9511024.

${ }^{14}$ A. S. Cattaneo, "Teorie topologiche di tipo BF ed invarianti dei nodi," Ph.D. thesis, Milan University, 1995 (available at $\mathrm{ftp} / / / \mathrm{pctheor}$.uni.mi.astro.it/pub/tesi.ps).

${ }^{15}$ M. Blau and G. Thompson, "Topological gauge theories of antisymmetric tensor fields," Ann. Phys. 205, 130-172 (1991).

${ }^{16}$ D. Birmingham, M. Blau, M. Rakowski, and G. Thompson, "Topological field theory," Phys. Rep. 209, 129-340 (1991).

${ }^{17}$ V. A. Vassiliev, "Cohomology of knot spaces" in Theory of Singularities and Its Applications, edited by V. Arnold (American Mathematical Society Providence, RI, 1990).

${ }^{18}$ E. Guadagnini, M. Martellini, and M. Mintchev, "Chern-Simons model and new relations between the HOMFLY coefficients," Phys. Lett. B 228, 489-494 (1989).

${ }^{19}$ D. Bar-Natan, "Perturbative aspects of the Chern-Simons field theory," Ph.D. thesis, Princeton University, 1991; "Perturbative Chern-Simons Theory," to appear in J. Knot Theory Ramifications.

${ }^{20}$ E. Witten, “Topological quantum field theory," Commun. Math. Phys. 117, 353-386 (1988).

${ }^{21}$ L. Rozansky, "Higher order terms in the Melvin-Morton expansion of the colored Jones polynomial," q-alg/9601009. 